This is an electronic reprint of the original article. This reprint may differ from the original in pagination and typographic detail.

Author(s): Tian, Yaolan; Isotalo, Tero; Konttinen, Mikko P.; Li, Jiawei; Heiskanen, Samuli; Geng, Zhuoran; Maasilta, Ilari

Title: Intregrating metallic wiring with three-dimensional polystyrene colloidal crystals using electron-beam lithography and three-dimensional laser lithography

Year: $\quad 2017$

Version:

Please cite the original version:

Tian, Y., Isotalo, T., Konttinen, M. P., Li, J., Heiskanen, S., Geng, Z., \& Maasilta, I. (2017). Intregrating metallic wiring with three-dimensional polystyrene colloidal crystals using electron-beam lithography and three-dimensional laser lithography. Journal of Physics D: Applied Physics, 50(5), Article 055302.

https://doi.org/10.1088/1361-6463/aa5004

All material supplied via JYX is protected by copyright and other intellectual property rights, and duplication or sale of all or part of any of the repository collections is not permitted, except that material may be duplicated by you for your research use or educational purposes in electronic or print form. You must obtain permission for any other use. Electronic or print copies may not be offered, whether for sale or otherwise to anyone who is not an authorised user. 


\title{
Intregrating metallic wiring with three-dimensional polystyrene colloidal crystals using electron-beam lithography and three-dimensional laser lithography
}

\author{
Yaolan Tian, Tero J. Isotalo†, Mikko P. Konttinen, Jiawei Li, \\ Samuli Heiskanen, Zhuoran Geng and Ilari J. Maasilta
}

E-mail: maasilta@jyu.fi

Nanoscience Center, Department of Physics, University of Jyvaskyla, P.O. Box 35, FI-40014 Jyväskylä, Finland

\begin{abstract}
We demonstrate a method to fabricate narrow, down to a few micron wide metallic leads on top of a three-dimensional colloidal crystal self-assembled from polystyrene (PS) nanospheres of diameter $260 \mathrm{~nm}$, using electron-beam lithography. This fabrication is not straightforward due to the fact that PS nanospheres cannot usually survive the harsh chemical treatments required in the development and lift-off steps of electron-beam lithography. We solve this problem by increasing the chemical resistance of the PS nanospheres using an additional electron-beam irradiation step, which allows the spheres to retain their shape and their self-assembled structure, even after baking to a temperature of 160 degrees $\mathrm{C}$, the exposure to the resist developer and the exposure to acetone, all of which are required for the electron-beam lithography step. Moreover, we show that by depositing an aluminum oxide capping layer on top of the colloidal crystal after the e-beam irradiation, the surface is smooth enough so that continuous metal wiring can be deposited by the electron-beam lithography. Finally, we also demonstrate a way to self-assemble PS colloidal crystals into a microscale container, which was fabricated using direct-write three-dimensional laser-lithography. Metallic wiring was also successfully integrated with the combination of a container structure and a PS colloidal crystal. Our goal is to make a device for studies of thermal transport in 3D phononic crystals, but other phononic or photonic crystal applications could also be envisioned. Keywords: self-assembly, colloidal crystal, cross-linking, electron-beam lithography, three-dimensional lithography, phononic crystal, photonic crystal
\end{abstract}

PACS numbers: 81.16.Dn, 81.16.Nd, 82.70.Dd, 65.80.-g

$\dagger$ Present address: Murata Electronics Oy 


\section{Introduction}

Colloidal crystallization of mono-disperse sub-micron spherical particles has been used successfully for years in the fabrication of three-dimensional (3D) photonic crystals $[1,2]$ for a wide variety of applications [3, 4, 5, 6]. Another emerging application for 3D colloidal crystals is the engineering of phonon band-structures in phononic crystals $(\mathrm{PnC})$, which have potential applications for example in engineering thermal

conductance $[7,8]$ and controlling hypersonic elastic waves $[6,9]$. So far, however, most of the work of the thermal conductance engineering has only been performed in lower dimensionalities, focusing mostly on the 2D hole array geometry either at room temperature $[10,11,12]$ or at low-temperatures $[13,14,15,16]$. One reason for this is that to perform thermal conductance experiments, metallic heater and thermometer structures are typically needed, and these structures need to be integrated with the actual phononic crystal structure. This wiring fabrication is typically done by lithographic means, and it is much easier to do this extra lithography step for $2 \mathrm{D}$ phononic crystal structures.

Integrating the metallic heaters and thermometers onto a 3D colloidal crystal surface poses several fabrication challenges, some of which we address in this study. Specifically, in this study we solve the following three problems: (i) Degradation and destruction of polymer colloidal particles due to harsh chemicals and heat, (ii) Deposition of continuous wires on the rough colloidal crystal surfaces, and (iii) Routing of the wiring onto a single crystalline domain of the colloidal crystal film. The first problem is especially severe for polymer colloids, such as polystyrene (PS), which are chemically close to the resists used in photo- and electron-beam lithography.

The first problem is solved by a highly controllable and simple method for protecting the PS spheres from chemical attack and melting, by cross-linking the PS spheres using high doses of electron-beam irradiation $[17,18]$. As this irradiation is performed in an electron-beam lithography system, the irradiation area can be controlled to be an arbitrary shape, with minimum lateral dimensions limited by the typical e-beam lithography resolution limits to below $100 \mathrm{~nm}$ resolution. Combined with the deposition of an aluminum oxide (AlOx) capping layer to smooth the rough colloidal crystal surface, we successfully fabricated lithographically patterned micron-scale narrow metallic wires on top of polystyrene colloidal crystals with a particle diameter $260 \mathrm{~nm}$. Finally, we demonstrate a novel way to fabricate a template for the colloidal crystal in the shape of a box from a photosensitive polymer, which was patterned directly using 3D laser writing and two-photon polymerization [19]. PS colloidal crystals were deposited inside this template box in such a way that metal leads for thermometry and heaters can be routed onto a thick $(\sim 10-20 \mu \mathrm{m})$ colloidal crystal domain from the surface of the substrate, without having to cross any cracks in the crystals that typically are generated in the vertical deposition self-assembly process of the colloidal crystal $[20,1,21,22,23]$. 


\section{Self-assembly of polystyrene colloidal crystals}

We have produced self-assembled colloidal crystals from polystyrene (PS) nanospheres of diameter $260 \mathrm{~nm}$ on silicon substrates. We used the vertical deposition method $[1,21,22,23]$, where the substrate was dipped into a 1-10\% aqueous solution of PS spheres, and was withdrawn slowly with speed typically ranging between $0.01-0.04$ $\mathrm{mm} / \mathrm{min}[20]$. The colloidal crystal film thickness and domain size could be controlled with the dipping speed and solution concentration, reaching values up to $20 \mu \mathrm{m}$ for the thickness and $\sim 300 \mu \mathrm{m}$ for both the vertical and horizontal directions of the domain size [20]. By domains we mean regions of the crystal surrounded by cracks that form in the drying process. These cracks are of course a serious issue for further lithographic patterning of wires on top, as they can be several micrometers wide.

The substrates used in most of the experiments were single crystal silicon chips, with a silicon nitride $\left(\mathrm{SiN}_{x}\right)$ insulator layer, and a thin layer of titanium oxide $\left(\mathrm{TiO}_{x}\right)$ approximately $10 \mathrm{~nm}$ thick on top of the $\mathrm{SiN}_{x}$, deposited by electron beam evaporation of $\mathrm{Ti}$ and room temperature oxidization in a vacuum chamber at 100 mbar of oxygen pressure for 2 minutes. The $\mathrm{TiO}_{x}$ layer provided a hydrophilic surface for improved wetting by the nano-sphere solution [24]. In the experiments with fabricated polymer template boxes, glass slides were used as the substrate, because the laser writing was performed through the substrate.

\section{Controlling the chemical resistance of a polystyrene colloidal crystal by electron-beam irradiation}

Many of the processes used in standard lithographic techniques involved are known to partially damage or completely destroy PS spheres. Specifically, the baking of electronbeam resists occurs at temperatures above the normal glass transition of polystyrene, thus destroying the spherical shape. Additionally, the acetone used in the standard lift-off of evaporated metals will very quickly dissolve polystyrene.

Here, we examine how electron-beam exposure affects the solubility and glass transition of colloidal crystals of $260 \mathrm{~nm}$ diameter PS spheres. It is known that UV [25, 26, 27] and e-beam exposure [17, 28] cause cross-linking in polystyrene, increasing the glass transition and chemical resistance $[17,18]$. E-beam cross-linking was chosen here over UV for a couple of reasons: electron beams are easily controlled for patterning without masks, and their penetration into the polymer layers is stronger that that of UV. This is very important in our application, where 10-20 $\mu \mathrm{m}$ thick colloidal crystals need to be cross-linked. UV light will not penetrate deep enough in this case.

\subsection{Process steps for the e-beam irradiation studies}

The process of deposition of the PS spheres and their exposure by an electron beam is schematically shown in Fig. 1 (a). 
(a) substrate

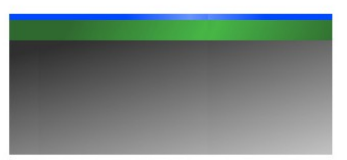

PS spheres



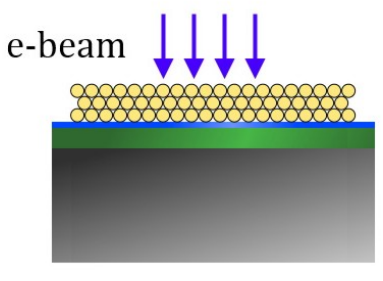

acetone

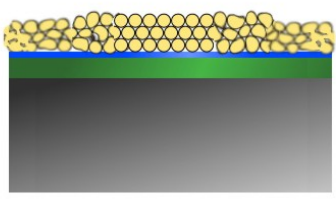

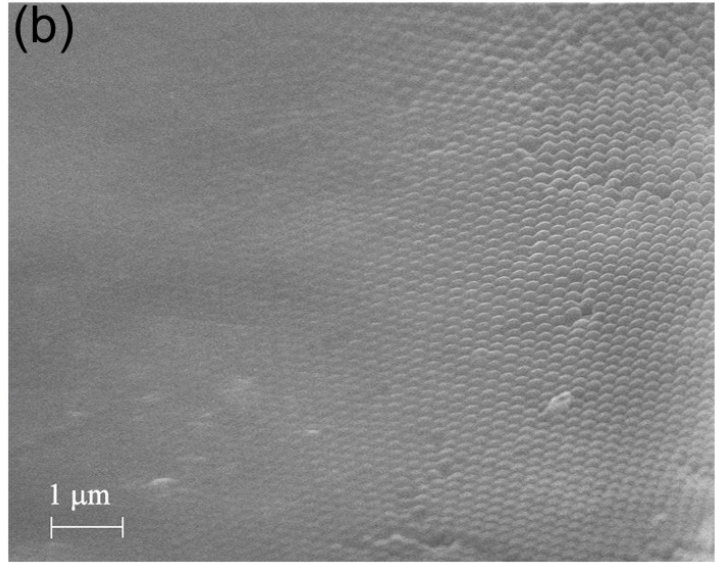

Figure 1: (a) The process steps for electron beam hardening of PS sphere colloidal crystals. Hydrophilic substrate is coated by spheres with vertical dipping, PS spheres are selectively cross-linked by an electron beam exposure, then sample is submerged in acetone for 5 minutes, to check the solubility. (b) An SEM micrograph of a vertical edge of an e-beam exposure area, showing a gradient of dissolution after an immersion into acetone.

A Si substrate with a TiOx surface layer ( with a colloidal crystal of $260 \mathrm{~nm}$ diameter PS spheres by vertical deposition [20], with a speed $0.04 \mathrm{~mm} / \mathrm{min}$ from a concentration of $5 \%$, resulting in a few layer thick colloidal crystal. The electron beam exposure was carried out in vacuum, using a JEOL JSM-840A scanning electron microscope operated at an acceleration voltage of $30 \mathrm{kV}$. All exposures were performed at $600 \mathrm{X}$ magnification, resulting in exposed areas with dimensions $230 \mu \mathrm{m} \times 160 \mu \mathrm{m}$. The beam current was varied from $75 \mathrm{pA}$ to $0.4 \mu \mathrm{A}$, with the exposure time kept constant at 5 minutes. Thus, the area dose ranged from $60 \mu \mathrm{C} / \mathrm{cm}^{2}$ to $326000 \mu \mathrm{C} / \mathrm{cm}^{2}$. Exposed samples were then immersed in acetone for several minutes. For high enough doses, the spheres in the exposed areas were seen to be protected from dissolution in acetone, while in the surrounding areas they were dissolved and formed a bulk PS film after drying, as seen in Figure 1 (b). There is also a clear, continuous gradient of the deformation of the spheres around the edge of the irradiation area, several microns long.

\subsection{Results on the increase of chemical resistivity of the PS colloidal crystals by electron-beam irradiation}

The samples treated by e-beam cross-linking were inspected by scanning electron microscopy (SEM). A large, continuous range of solubility in acetone was seen as a function of the exposure dose. That is already evident in Fig. Figure 1 (b) as a spatial

gradient of the dissolution around the edge of the irradiation area. SEM images were taken of each sample at several locations to ensure the results were consistent across the surface. Qualitative characterization was made by observing the degree to which the 
PS spheres were dissolved by the acetone at a given exposure dose.

We discovered that below the exposure dose $\sim 2300 \mu \mathrm{C} / \mathrm{cm}^{2}$, there was a range of doses where the solubility was highly controllable (Figs. 2 and 3). A lower critical dose for inducing chemical resistance to acetone was found to lie between $60 \mu \mathrm{C} / \mathrm{cm}^{2}$ and $170 \mu \mathrm{C} / \mathrm{cm}^{2}$, consistent with previous studies on bulk PS films [17]. The 1-2 layer thick samples exposed to $60 \mu \mathrm{C} / \mathrm{cm}^{2}$ are seen in Figure 2 (a) (left) to dissolve completely in acetone, while those exposed with $170 \mu \mathrm{C} / \mathrm{cm}^{2}$ Figure 2 (a) (right) exhibited some signs of spherical structures remaining within the dissolved mass.
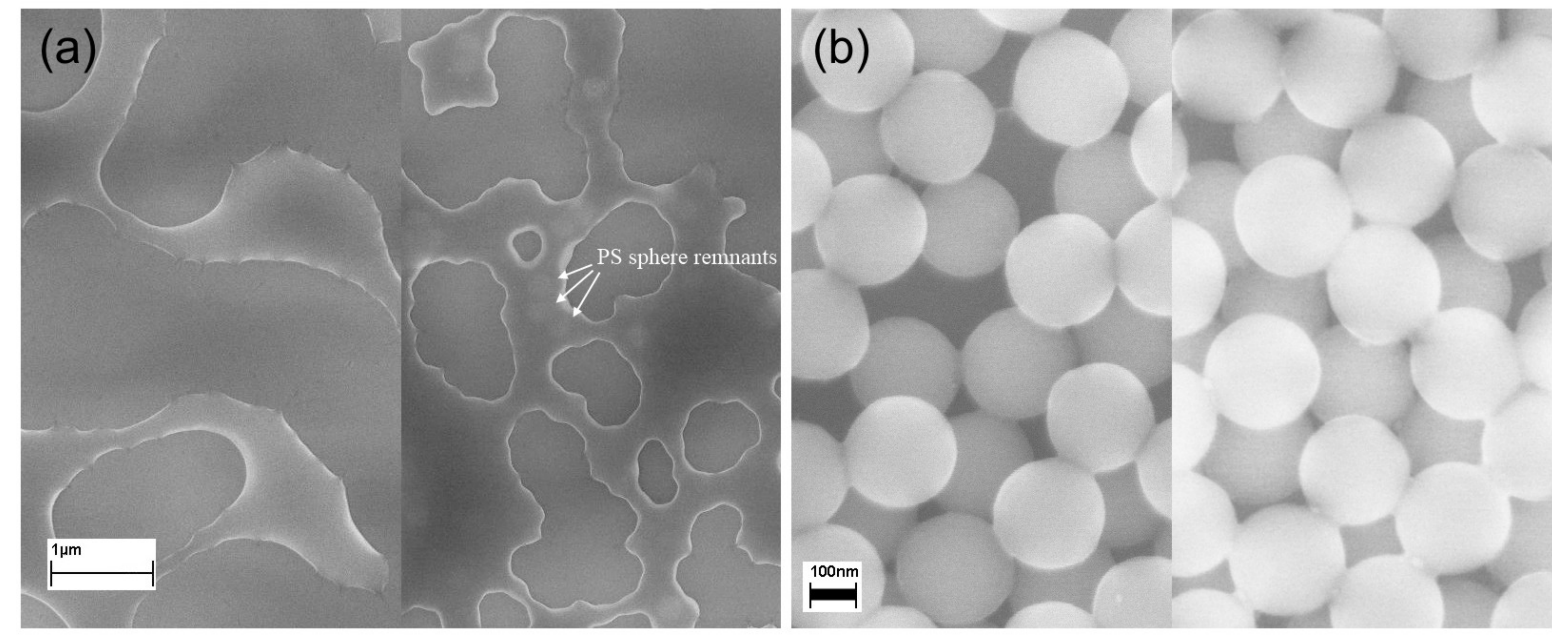

Figure 2: (a) PS spheres after e-beam exposure and acetone bath are shown for a dose of $60 \mu \mathrm{C} / \mathrm{cm}^{2}$ (left) and $170 \mu \mathrm{C} / \mathrm{cm}^{2}$ (right). Some PS sphere remnants can be seen within the dissolved mass on the right, while spheres are completely dissolved at the lower exposure dose on the left. Scale bar is $1 \mu \mathrm{m}$. (b) PS spheres after e-beam exposure and acetone bath are shown for a dose of $2285 \mu \mathrm{C} / \mathrm{cm}^{2}$ (left) and $36850 \mu \mathrm{C} / \mathrm{cm}^{2}$ (right). Solubility in both cases is negligible. Scale bar in black is $100 \mathrm{~nm}$.

At a dose of $\sim 2200 \mu \mathrm{C} / \mathrm{cm}^{2}$ and above, the solubility of the PS spheres in acetone was negligible. Figure 2 (b) shows a comparison between PS spheres given a dose of $2285 \mu \mathrm{C} / \mathrm{cm}^{2}$ (left) and $36850 \mu \mathrm{C} / \mathrm{cm}^{2}$ (right), exposed to acetone for 5 minutes. The spheres have clearly retained their original shape to a high degree, and we can state with confidence that both samples are effectively resistant against solubility.

Based on Fig. 2, it is worth while to study in more detail what happens with doses between $170 \mu \mathrm{C} / \mathrm{cm}^{2}$ and $2285 \mu \mathrm{C} / \mathrm{cm}^{2}$. Figure 3 shows the SEM images of samples exposed in this intermediate regime. We see that increasing the dose resulted in a controllable and gradual increase of the chemical resistivity in acetone. This range is large enough to allow precise tuning of the selected dose. There is even evidence that one has some control over the formation of narrow neck connections between individual PS spheres (at doses between $475 \mu \mathrm{C} / \mathrm{cm}^{2}$ and $1225 \mu \mathrm{C} / \mathrm{cm}^{2}$ ). The narrow necks can be extremely narrow, for example for the dose $1225 \mu \mathrm{C} / \mathrm{cm}^{2}$ they are only $\sim 5 \mathrm{~nm}$ wide.

Although the acetone resistance studies in Figs. $1-3$ were performed with thin 


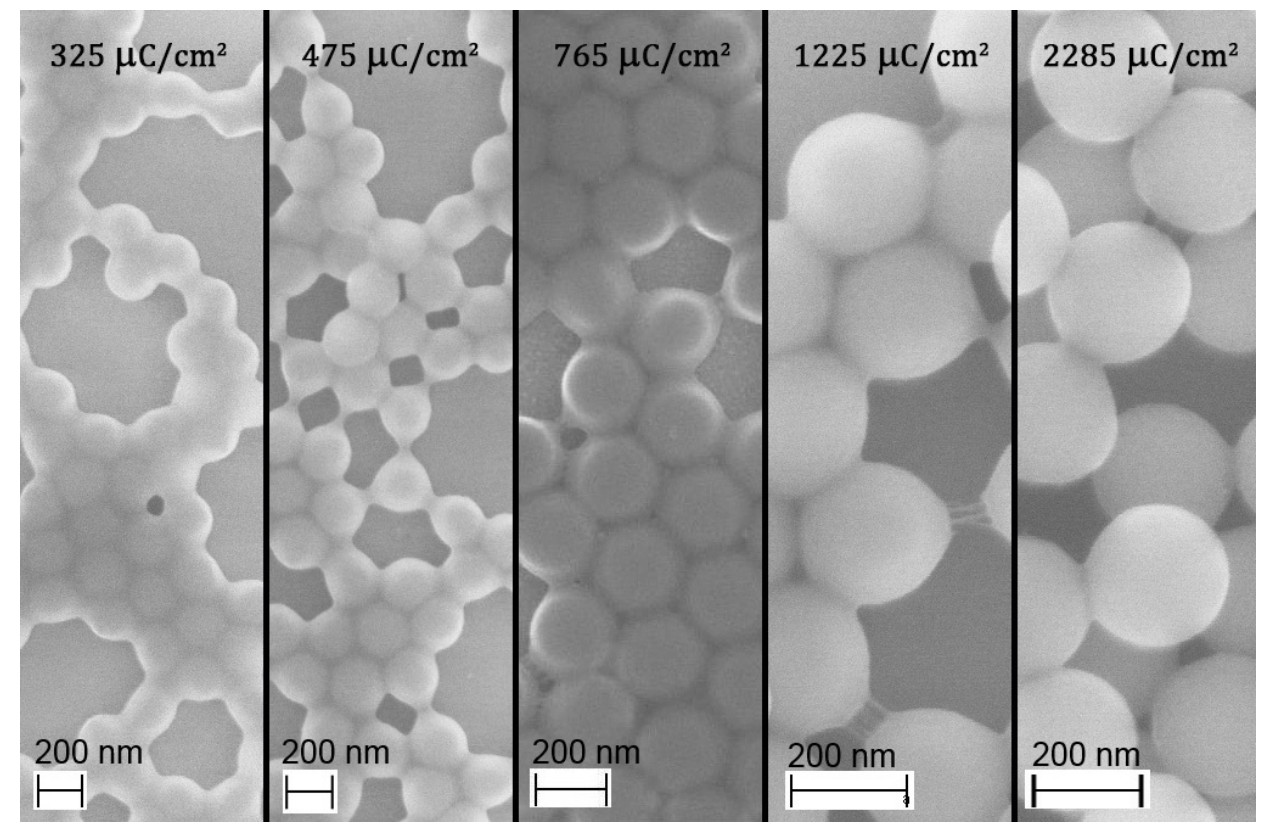

Figure 3: PS spheres after e-beam exposure and acetone bath are shown for doses ranging from $325 \mu \mathrm{C} / \mathrm{cm}^{2}$ to $2285 \mu \mathrm{C} / \mathrm{cm}^{2}$ (left to right). A highly tunable resistance to dissolution in acetone is seen.

1-2 layer colloidal crystals, we have also performed successful e-beam irradiations with doses above $\sim 1000 \mu \mathrm{C} / \mathrm{cm}^{2}$ on much thicker crystals, up to 20 layers thick $(\sim 4 \mu \mathrm{m})$. For crystals thicker than that, we typically observed that the whole crystal peeled off from the substrate after irradiation. However, later in section 5 we show a templating method, with which the thickness could be increased to $\sim 10 \mu \mathrm{m}$ without peel-off after irradiation.

\section{Lithography on colloidal crystal surfaces}

Lithographic testing was performed using two types of samples, both given cross-linking area doses of $\sim 24000 \mu \mathrm{C} / \mathrm{cm}^{2}$ and immersed first in acetone for 5 minutes to check that no dissolution resulted. One set of samples were then spin coated with three layers of electron-beam resists baked at $70{ }^{\circ} \mathrm{C}$ for 5 minutes after each layer. The resist layers were spun coated onto the colloidal crystal, with spin speeds corresponding to nominal thicknesses on bare substrates of one $400 \mathrm{~nm}$ layer of copolymer poly(methyl methacrylate-methacrylic acid) [P(MMA-MAA)], and two layers of $200 \mathrm{~nm}$ poly(methyl methacrylate) (PMMA), giving a nominal total thickness $800 \mathrm{~nm}$. This type of multilayer resist was used to help with the lift-off step, as it created an undercut structure. Another colloidal crystal sample set was first coated with $\mathrm{AlO}_{x}$ layer of thickness $\sim 100 \mathrm{~nm}$ by electron beam evaporation, using a rotating sample stage and angle evaporation from 70 degrees, before the spin coating with the three resist layers. A schematic for the second method using the AlOx capping is shown in Figure 4. After 

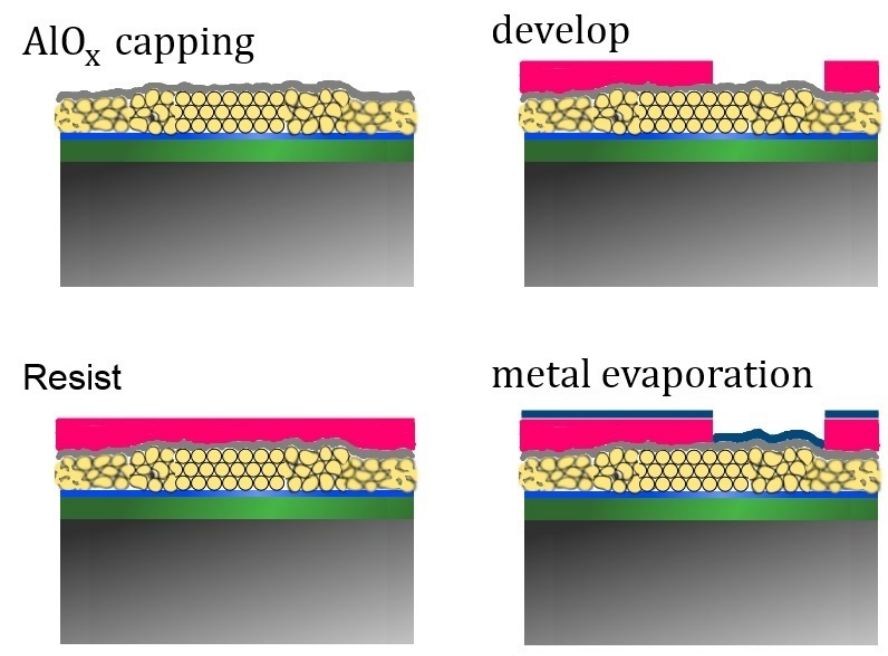

metal evaporation
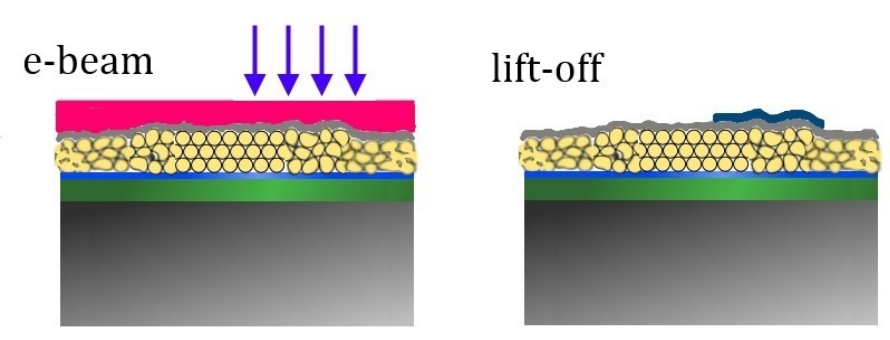

Figure 4: Process steps are shown for lithography on PS sphere colloidal crystal surface. $\mathrm{AlO}_{x}$ capping layer is e-beam evaporated on colloidal crystal sample, PMMA+P(MMAMAA) three-layer resist is spin coated, e-beam pattern is exposed and then developed, metal is evaporated and lift-off is performed.

the e-beam resist coating, a lithographic pattern was exposed using a Raith e-LiNE tool with a $20 \mathrm{kV}$ electron beam, developed in MIBK:IPA (1:3) solution for 60 seconds, rinsed in IPA for 15 - 30 seconds and dried under nitrogen flow. The developed samples were then coated with $40 \mathrm{~nm}$ to $80 \mathrm{~nm}$ of aluminum (Al) by electron beam evaporation in ultra-high vacuum, evaporated perpendicular to the substrate, and finally the metal liftoff was performed by placing samples in cold acetone for 30 minutes and then applying a mildly turbulent flow from a syringe.

For lithographic testing purposes, simple aluminum wires were deposited by the above electron beam evaporation and lift-off steps, with patterns of varying line width and typical film thickess 40-80 $\mathrm{nm}$.

It was found that lithography without the $\mathrm{AlO}_{x}$ capping layer was possible, but produced inconsistent results. It seemed that the thickness of e-beam resist was notably less on the colloidal crystal surface than on a plain surface. On the PS sphere surface, a minimum of three layers of electron-beam resist was necessary to cover the self-assembled crystal surface. This may be due to resist penetration into the spaces between spheres, as well as inhomogeneous spreading over different heights of the PS sphere structures. Even with the increased resist thickness, the sharpness of lithographic structures was 

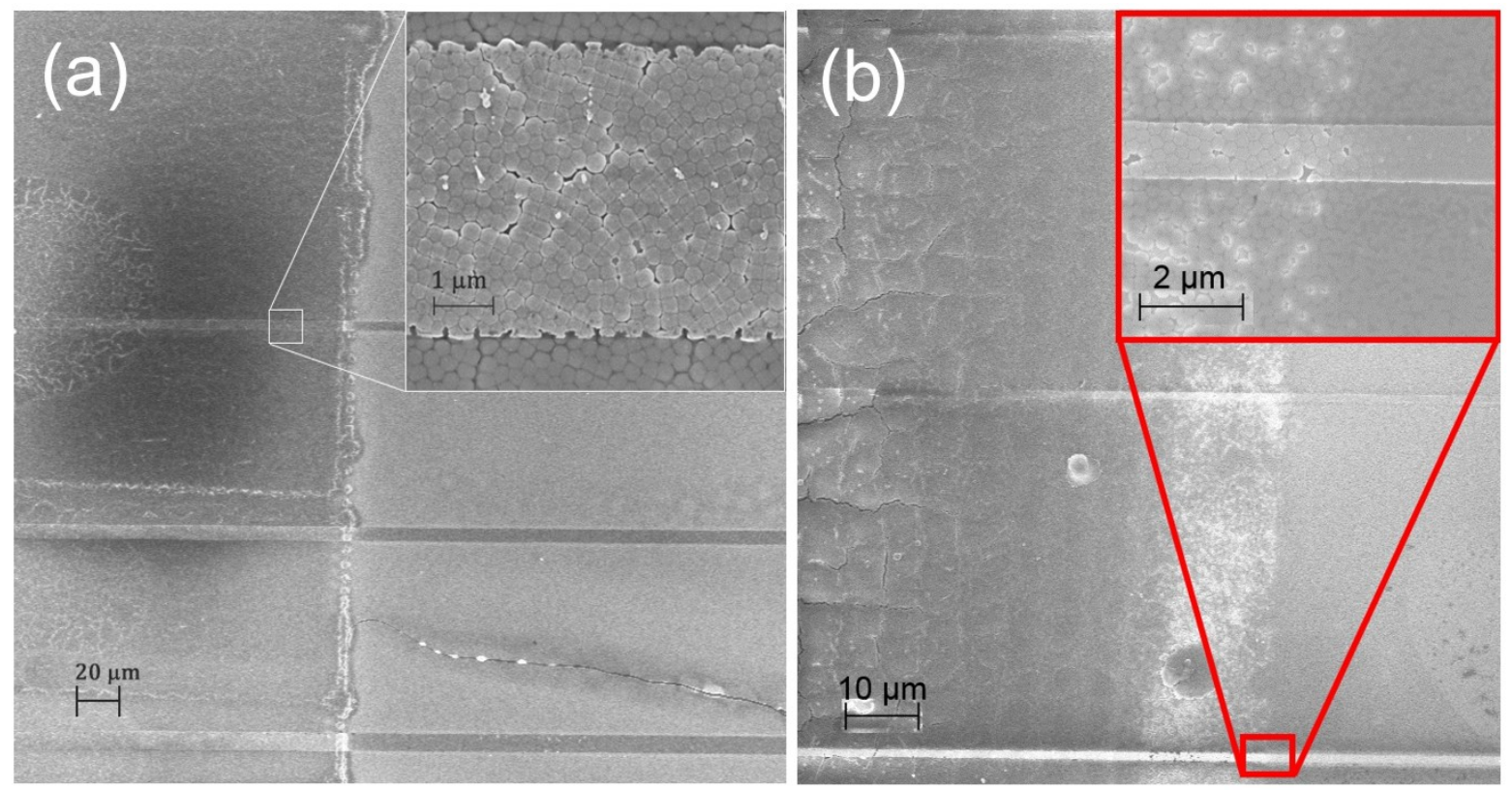

Figure 5: SEM imaging of $\mathrm{Al}$ wires deposited by electron beam evaporation is on top of a hardened PS sphere colloidal crystal with an $\mathrm{AlO}_{x}$ capping layer. (a) Wire widths ranging from 5 to $10 \mu \mathrm{m}$. (b) Wire widths around $1 \mu \mathrm{m}$. The roughness of the metal wire edge (insets) is seen to be less than the sphere size.

reduced compared to patterns made on plain substrates without PS spheres. Thus, a capping layer was needed to minimize the penetration of the resists into the crystal structure, and to smooth the top surface of the crystal.

Using a $100 \mathrm{~nm}$ thick evaporated $\mathrm{AlO}_{x}$ capping layer, we were able to produce continuous and conducting metal wires a few hundred $\mu \mathrm{m}$ in length and as narrow as one $\mu \mathrm{m}$ (Figure 5). Roughness at the edges of the wires, as seen in the insets in Figure 5, was found to be low enough to suggest the possibility of producing wires approaching the diameter of PS spheres. While sub-micron wires have also been fabricated, there are still issues with wire continuity and thus, electrical conductivity. Further development in our lithographic processing is expected to enable the fabrication of $\sim 500 \mathrm{~nm}$ conducting metal wires on the colloidal crystal surfaces.

\section{Microscale template structure for colloidal crystals using 3D lithography}

So far we have only discussed results where the PS colloidal crystals were deposited on large areas everywhere on the substrate. This may not be practical for eventual devices, where we would like to control the placement of the crystals on the chip, and also have the metal wiring routed from the surface of a colloidal crystal to an area on the chip without the colloids, where wire bonding would be performed (wire bonding is not easily possible on the soft PS colloidal film). There are several methods to lithographically control the deposition of colloidal crystals, such as by engineering contrast in the hydrophilicity of 
the substrate $[29,22]$ or the local evaporation rate [30], or by a simple spatial confinement by patterned topographic structures $[31,32,33,34,20]$. Here we follow the route of topographic patterning, but in contrast to many previous studies, we want to control very thick colloidal crystals of the thickness range $10-20 \mu \mathrm{m}$. This is about the same thickness as in Refs. [31] and [32], but in contrast to Ref. [31] and our own previous work [20], we do not use deep etching in Si, but want to pattern a negative photoresist on glass substrates as an additive process, as in Ref. [32].

2D photolithographic patterning of negative photoresists such as SU-8, on the other hand, is naturally limited to simple 2D structures [32]. Here, we use three-dimensional patterning to create more complex, fully $3 \mathrm{D}$ shapes. This is possible with the help of direct laser-write lithography using two-photon absorption [35], where infrared light at $780 \mathrm{~nm}$ is focussed tightly with the help of a microscope. Only at the very focus of the beam two-photon absorption takes place, where the simultaneous absorption of two IR photons can excite the photoresist the same way as a single UV photon at $390 \mathrm{~nm}$ [19]. The patterning was performed with the instrument by Nanoscribe GmbH using the standard mode, where the laser beam was focused through a glass substrate onto a liquid polymer resist drop on the opposite surface.

The design of the structure is shown in Figure 6. It is a square shaped box of height $10 \mu \mathrm{m}$, with inside, "container area" dimensions $100 \mu \mathrm{m} \times 100 \mu \mathrm{m}$, surrounded by walls of width $25 \mu \mathrm{m}$ which are themselves made from hollow beam structures for structural rigidity and ease of fabrication. Outside walls connect to sloped regions of angle 45 degrees, the purposes of which are to i) attach the box structure more securely to the substrate, and ii) to allow the eventual routing of metal wiring from the substrate surface, where the bonding pads will be located, to the top of the box structure. The SEM image shows that some distortion of the shape is observable, due to mechanical forces created by the shrinking of the IPL780 photoresist (Nanoscribe GmbH) during development. This slight distortion can, however, be improved by design improvements in the future.

With the support box available, we deposited PS colloidal crystals into them by the vertical dipping technique, using $1 \%$ PS nano-sphere colloidal solution at a dipping speed of $0.01 \mathrm{~mm} / \mathrm{min}$. Figure 7 shows a SEM micrograph which shows part of the 3D box structure (top view) after colloidal crystal deposition. We see that crystals have been deposited inside the box, as hoped for, and the photoresist walls and slopes have no PS nanospheres on them. This is quite useful as further lithography of metal lines on the bare box structure is easier.

In addition, we make two other useful observations from Figure 7: i) Below the box structure, there is a region of bare glass substrate visible. It seems that the box structure prevented the deposition of colloidal crystal there. This kind of region can thus be used for the bonding pads and wiring, climbing over the slopes and finally onto the colloidal crystal surfaces inside the box. ii) The colloidal crystals can be in contact with the box inside wall (inset), without a significant crack. This is in contrast with our earlier work with etched silicon boxes [20], where cracks were always observed between 

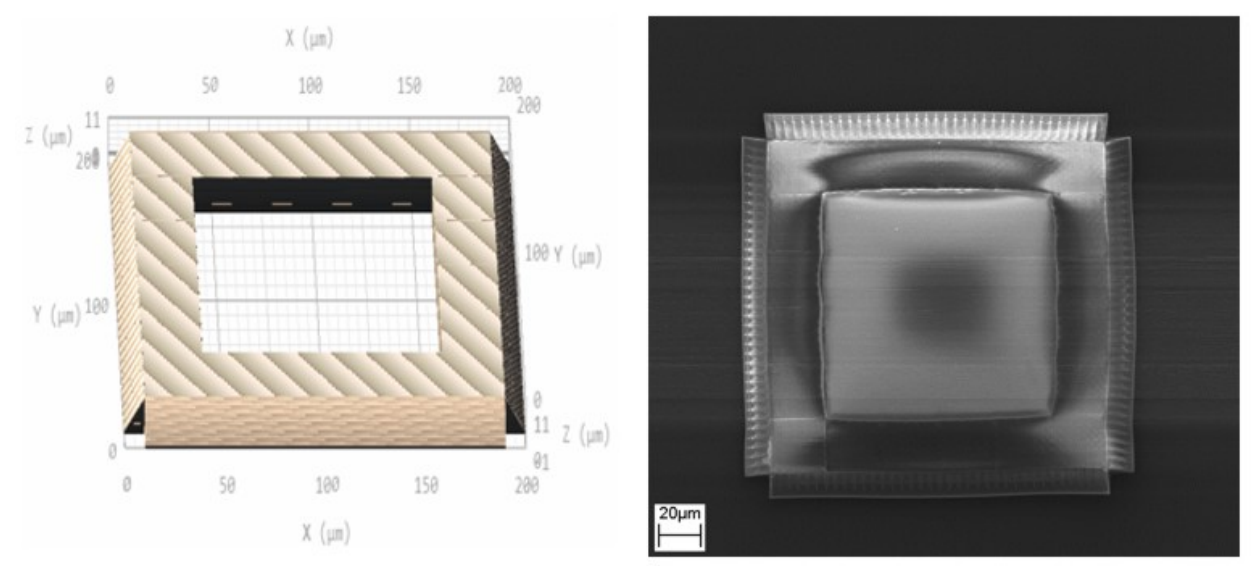

Figure 6: The design of the 3D resist box. Left: schematic design, with walls and slopes surrounding outside walls. Right: SEM micrograph of a fabricated structure from top view.



Figure 7: SEM image of PS colloidal crystals deposited on the 3D lithographically fabricated photoresist box structure. Inset: a zoom in of an area at the inside edge of the box.

the box wall and colloidal crystal. However, cracks were clearly observable inside the box structure. It is of course possible to reduce the lateral size of the box in the future to such a degree that only a single domain would fit inside.

To further prove that the routing of wires from a polymer box structure onto the colloidal crystal is possible, we also fabricated another device where $3 \mu \mathrm{m}$ wide $\mathrm{Al}$ wiring of thickness $100 \mathrm{~nm}$ was deposited on a box structure of size $50 \times 50 \mu \mathrm{m}$, containing a 10 $\mu \mathrm{m}$ thick PS colloidal crystal deposited by vertical dipping solely into the box container area, shown in a SEM micrograph in Fig. 8. For the fabrication of the structure in Fig. 8, all the techniques described earlier had to be combined, i.e. fabrication of a photoresist 


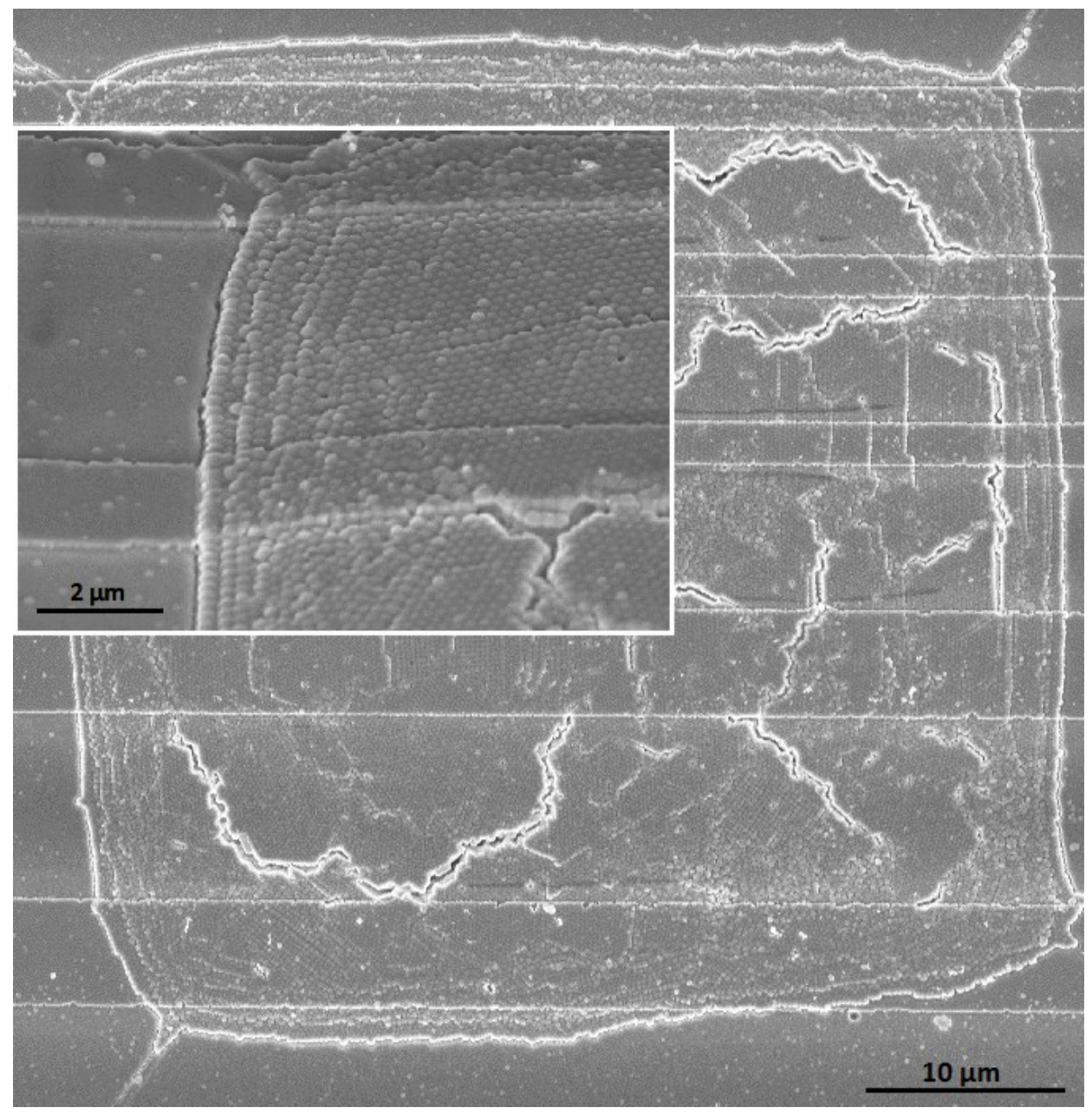

Figure 8: SEM image of $2 \mu \mathrm{m}$ wide $\mathrm{Al}$ wiring integrated with $10 \mu \mathrm{m}$ thick PS colloidal crystals which were deposited on a photoresist box structure of size $50 \times 50 \mu \mathrm{m}$. The inset shows a zoomed-in image at around the edge of the box inside wall.

box structure, vertical dipping for colloidal crystal self-assembly, e-beam hardening of the PS crystal, AlOx capping layer deposition, and finally e-beam lithography of the $\mathrm{Al}$ line features using lift-off.

\section{Conclusions}

In conclusion, we have presented a versatile method for the fabrication of metallic wiring on top of polystyrene colloidal crystals, which will in the future allow for electronic devices to be processed on top of the crystals. The critical steps developed here were: i) Lithographically controlled, electron-beam induced cross-linking, to harden polystyrene colloidal crystals so that they can withstand the elevated temperatures and harsh chemicals used in electron-beam lithography. This allows standard lithographic processes to be used on top of the self-assembled polystyrene nanosphere colloidal crystals. ii) Deposition of micron-scale narrow metal wiring on top of the colloidal 
crystals, and iii) Templated deposition of the colloidal crystals into pre-defined areas that can allow wiring to come from the substrate surface onto the crystals. These truly 3D shaped templates were patterned from a photoresist, using direct laser writing and twophoton absorption, which is thus a fully three-dimensional lithographic technique. The developed techniques will be used in the future to integrate heaters and thermometers on colloidal structures, which is critical for example for the future studies of phonon transport in three-dimensional phononic crystal structures. In addition, the methods developed here can be utilized in devices where complex metal structures need to be integrated with a 3D photonic crystal.

\section{Acknowledgments}

This research was supported by the Academy of Finland Project No. 260880. We thank Onni-Pekka Häkkinen and Emmi Kirjanen for fabrication assistance.

\section{References}

[1] P. Jiang, J. F. Bertone, K. S. Hwang, and V. L. Colvin. Single-crystal colloidal multilayers of controlled thickness. Chemistry of Materials, 11(8):2132-2140, 1999.

[2] Yurii A. Vlasov, Xiang-Zheng Bo, James C. Sturm, and David J. Norris. On-chip natural assembly of silicon photonic bandgap crystals. Nature, 414:289 - 293, 2001.

[3] J D Joannopoulos, Pierre R Villeneuve, and Shanhui Fan. Photonic crystals: putting a new twist on light. Nature, 386(6621):143-149, 1997.

[4] Younan Xia, Byron Gates, Yadong Yin, and Yu Lu. Monodispersed colloidal spheres: Old materials with new applications. Advanced Materials, 12:693-713, 2000.

[5] Juan F. Galisteo-Lopez, Marta Ibisate, Riccardo Sapienza, Luis S. Froufe-Perez, Alvaro Blanco, and Cefe Lopez. Self-assembled photonic structures. Advanced Materials, 23:30-69, 2011.

[6] Jae-Hwang Lee, Cheong Yang Koh, Jonathan P. Singer, Seog-Jin Jeon, Martin Maldovan, Ori Stein, and Edwin L. Thomas. 25th anniversary article: Ordered polymer structures for the engineering of photons and phonons. Advanced Materials, 26(4):532-569, 2014.

[7] T. J. Isotalo, Y. L. Tian, and I. J. Maasilta. Fabrication and modelling of three-dimensional sub-kelvin phononic crystals. Journal of Physics: Conference Series, 400:052007, 2012.

[8] Martin Maldovan. Sound and heat revolutions in phononics. Nature (London), 503:209-217, 2013.

[9] Wei Cheng, Jianjun Wang, Ulrich Jonas, George Fytas, and Nikolaos Stefanou. Observation and tuning of hypersonic bandgaps in colloidal crystals. Nature Materials, 5:830 - 836, 2006.

[10] J. Tang, H.-T. Wang, D.H. Lee, M. Fardy, Z. Huo, T.P. Russell, and P. Yang. Holey silicon as an efficient thermoelectric material. Nano Lett., 10:4279-4283, 2010.

[11] J.-K. Yu, S. Mitrovic, D. Tham, J. Varghese, and J.R. Heath. Reduction of thermal conductivity in phononic nanomesh structures. Nat. Nanotech., 5:718-721, 2010.

[12] S. Alaie, D.F. Goettler, M. Su, Z.C. Leseman, C.M. Reinke, and I. El-Kady. Thermal transport in phononic crystals and the observation of coherent phonon scattering at room temperature. Nat. Commun., 6:7228, 2015.

[13] Nobuyuki Zen, Tuomas A. Puurtinen, Tero J. Isotalo, Saumyadip Chaudhuri, and Ilari J. Maasilta. Engineering low-temperature thermal conductance using a two-dimensional phononic crystal. Nature Communications, 5:3435, 2014.

[14] Tuomas A. Puurtinen and Ilari J. Maasilta. Low-temperature coherent thermal conduction in thin phononic crystal membranes. Crystals, 6:72, 2016. 
[15] R. Anufriev, J. Maire, and M. Nomura. Reduction of thermal conductivity by surface scattering of phonons in periodic silicon nanostructures. Phys. Rev. B, 93:045411, 2016.

[16] R. Anufriev and M. Nomura. Reduction of thermal conductance by coherent phonon scattering in two-dimensional phononic crystals of different lattice types. Phys. Rev. B, 93:045410, 2016.

[17] Wolfram Schnabel and Hideto Sotobayashi. Polymers in electron beam and x-ray lithography. Progress in Polymer Science, 9(4):297 - 365, 1983.

[18] R. L. Clough. High energy radiation and polymers: A review of commercial processes and engineering applications. Nuclear Instruments and Methods in Physics Research B, 185:8-33, 2001.

[19] Markus Deubel, Georg von Freymann, Martin Wegener, Suresh Pereira, Kurt Busch, and Costas M. Soukoulis. Direct laser writing of three-dimensional photonic-crystal templates for telecommunications. Nature materials, 3:444-447, 2004.

[20] Tero J. Isotalo, Yao-Lan Tian, Mikko P. Konttinen, and Ilari J. Maasilta. Statistical characterization of self-assembled colloidal crystals bysingle-step vertical deposition. Colloids and Surfaces A: Physicochemical and Engineering Aspects, 443:164-170, 2014.

[21] Zhong-Ze Gu, Akira Fujishima, and Osamu Sato. Fabrication of high-quality opal films with controllable thickness. Chemistry of Materials, 14:760-765, 2013.

[22] Charles-Andre Fustin, Gunnar Glasser, Hans W. Spiess, and Ulrich Jonas. Parameters influencing the templated growth of colloidal crystals on chemically patterned substrates. Langmuir, 20:9114-9123, 2004.

[23] Robert G. Shimmin, Alexander J. DiMauro, and Paul V. Braun. Slow vertical deposition of colloidal crystals: A langmuir-blodgett process? Langmuir, 22(15):6507-6513, 2006.

[24] Subbian Karuppuchamy and Jae Mun Jeong. Super-hydrophilic amorphous titanium dioxide thin film deposited by cathodic electrodeposition. Materials Chemistry and Physics, 93(2-3):251 $254,2005$.

[25] R. Ranby and J. F. Rabek. Photodegradation, Photo-oxidation and photostabilization of polymers. John Wiley and Sons, New York, 1975.

[26] Lei Li, Caikang Chen, Aijuan Zhang, Xinyu Liu, Kun Cui, Jin Huang, Zhi Ma, and Zhaohui Han. Fabrication of robust honeycomb polymer films: A facile photochemical cross-linking process. Journal of Colloid and Interface Science, 331(2):446 - 452, 2009.

[27] Marta Palacios, Olga Garca, and Juan Rodrguez-Hernndez. Constructing robust and functional micropatterns on polystyrene surfaces by using deep uv irradiation. Langmuir, 29(8):2756-2763, 2013. PMID: 23363393.

[28] Siqi Ma, Celal Con, Mustafa Yavuz, and Bo Cui. Polystyrene negative resist for high-resolution electron beam lithography. Nanoscale Research Letters, 6(1):1-6, 2011.

[29] C.-A. Fustin, G. Glasser, H.W. Spiess, and U. Jonas. Site-selective growth of colloidal crystals with photonic properties on chemically patterned surfaces. Advanced Materials, 15(12):1025-1028, 2003.

[30] Daniel J. Harris, Hua Hu, Jacinta C. Conrad, and Jennifer A. Lewis. Patterning colloidal films via evaporative lithography. Phys. Rev. Lett., 98:148301, Apr 2007.

[31] P Ferrand, M J Minty, M Egen, J Ahopelto, R Zentel, S G Romanov, and C M Sotomayor Torres. Micromoulding of three-dimensional photonic crystals on silicon substrates. Nanotechnology, 14(2):323, 2003.

[32] Jian Xu, Eoin S. O'Keefe, and Carole C. Perry. Opaline films on patterned substrates by a simple self-assembly method. Materials Letters, 58(2728):3419 - 3423, 2004.

[33] Shih-Kai Wu, Tzu-Piao Tang, and Wenjea J. Tseng. Self-assembly of polystyrene microspheres within spatially confined rectangular microgrooves. Journal of Materials Science, 43(19):6453$6458,2008$.

[34] Lidiya Mishchenko, Benjamin Hatton, Mathias Kolle, and Joanna Aizenberg. Patterning hierarchy in direct and inverse opal crystals. Small, 8(12):1904-1911, 2012.

[35] Satoshi Kawata, Hong-Bo Sun, Tomokazu Tanaka, and Kenji Takada. Finer features for functional 

microdevices. Nature, 412:697-698, 2001. 